\title{
LTBP2 null mutations in an autosomal recessive ocular syndrome with megalocornea, spherophakia, and secondary glaucoma
}

\author{
Julie Désir ${ }^{\star, 1,2}$, Yves Sznajer ${ }^{2,3}$, Fanny Depasse ${ }^{4}$, Françoise Roulez ${ }^{5}$, Marc Schrooyen ${ }^{4}$, Françoise Meire ${ }^{4,5,6}$ and
} Marc Abramowicz ${ }^{1,2}$

The latent TGF $\beta$-binding proteins (LTBPs) and fibrillins are a superfamily of large, multidomain proteins with structural and TGF $\beta$-signalling roles in the extracellular matrix. Their importance is underscored by fibrillin-1 mutations responsible for Marfan syndrome, but their respective roles are still incompletely understood. We report here on two families where children from healthy, consanguineous parents, presented with megalocornea and impaired vision associated with small, round, dislocated lenses (microspherophakia and ectopia lentis) and myopia, as well as a high-arched palate, and, in older children, tall stature with an abnormally large arm span over body height ratio, that is, associated features of Marfan syndrome. Glaucoma was not present at birth, but was diagnosed in older children. Whole genome homozygosity mapping followed by candidate gene analysis identified homozygous truncating mutations of $L T B P 2$ gene in patients from both families. Fibroblast mRNA analysis was consistent with nonsense-mediated mRNA decay, with no evidence of mutated exon skipping. We conclude that biallelic null LTBP2 mutations cause the ocular phenotype in both families and could lead to Marfan-like features in older children. We suggest that intraocular pressures should be followed-up in young children with an ocular phenotype consisting of megalocornea, spherophakia and/or lens dislocation, and recommend LTBP2 gene analysis in these patients.

European Journal of Human Genetics (2010) 18, 761-767; doi:10.1038/ejhg.2010.11; published online 24 February 2010

Keywords: microspherophakia; megalocornea; Marfan

\section{INTRODUCTION}

Marfan syndrome (MFS, MIM 154700) is a connective tissue disorder with autosomal dominant inheritance. ${ }^{1}$ MFS typically affects the skeletal system with a disproportionate increase in the linear growth of long bones (tall stature with increased arm span and decreased upper-to-lower body ratio, arachnodactyly, dolichostenomelia, pectus deformity, and scoliosis) and joint hyperlaxity; the ocular system with ectopia lentis in $55 \%$ of affected $<18$ years, ${ }^{2}$ as well as microspherophakia (small round lens) and megalocornea (large cornea) in severe congenital forms; ${ }^{3,4}$ and the cardiovascular system with thick, elongated cardiac valves and progressive aortic root enlargement. Additional features of MFS include striae distensae of the skin, a decrease in muscle and fat mass, and a predisposition to spontaneous pneumothorax. Together with progressive aortic enlargement or rupture, these features indicate an increased elastolysis and defective tissue repair. ${ }^{5}$ MFS is caused by mutations in $F B N 1$, whose protein product fibrillin-1 (FBN1) is a major structural component of the microfibrils. ${ }^{6}$ Beyond the structural defect of microfibrils, however, abnormal signaling by growth factors belonging to the TGF $\beta$ family have an important role in MFS. ${ }^{7,8}$ An altered cellular behavior during development, associated with an increased TGF $\beta$ activity, is further supported by mouse models of MFS. ${ }^{9}$ Aortic aneurysm in a mouse model of MFS can be prevented by TGF $\beta$ antagonists such as a TGF $\beta$-neutralizing antibody or the angiotensin II type 1 receptor blocker, losartan. ${ }^{10}$

The TGF $\beta$ s are synthesized as prepropolypeptides, and processed in the Golgi apparatus into mature TGF $\beta$ tightly associated with its propeptide, the latency-associated peptide (LAP). In the extracellular matrix, the LAP.TGF $\beta$ complex is further covalently bound to a latent TGF $\beta$-binding protein (LTBP). ${ }^{11}$ The LTBPs are a family of proteins that show important structural homologies with the fibrillins. ${ }^{7}$ They are composed of a variety of repeated cystein-rich modules, including an 8-cysteine modular structure found only in fibrillins and LTBPs. ${ }^{12}$ Some, but not all, of the 8-cystein modules can mediate binding to the LAP.TGF $\beta 1$ complex. ${ }^{13,14}$ Although LTBP1, LTBP3, and to a lesser extent LTBP4 were reported to form a covalent complex with LAP.TGF $\beta 1$, LTBP2, and the fibrillins do not. ${ }^{15}$ In addition, the LTBPs and fibrillins contain at least one hybrid cystein-rich domain, which can mediate intermolecular disulfide bonding and may hence have an important role in microfibril assembly. ${ }^{16}$ LTBP1, and presumably other LTBP molecules, is transglutaminase crosslinked to the extracellular matrix via its amino-terminal region. ${ }^{7}$ This model allows for fine temporal and spatial regulation of TGF $\beta$ sequestering or release in the extracellular matrix. LTBP1, LTBP2, and LTBP4, interact through their carboxy-terminal region with FBN1., ${ }^{77}$ LTBP2 also

${ }^{1}$ IRIBHM, Université Libre de Bruxelles (ULB), Brussels, Belgium; ${ }^{2}$ Department of Medical Genetics, Hôpital Erasme-ULB, Brussels, Belgium; ${ }^{3}$ Clinical Genetics Unit, HUDERF-ULB, Brussels, Belgium; ${ }^{4}$ Department of Ophthalmology, Hôpital Erasme-ULB, Brussels, Belgium; ${ }^{5}$ Department of Ophthalmology, HUDERF-ULB, Brussels, Belgium; ${ }^{6}$ Department of Ophthalmology, University Hospital, Gent, Belgium

*Correspondence: Dr J Désir, Department of Medical Genetics, Hôpital Erasme-ULB, 808 Route de Lennik, B-1070 Brussels, Belgium. Tel: +32 2555 7076 ;

Fax: +32 2555 4212; E-mail: jdesir@ulb.ac.be

Received 24 August 2009; revised 5 January 2010; accepted 5 January 2010; published online 24 February 2010 
interacts through its amino-terminal domain with fibulin-5 suggesting a role for LTBP2 in the regulation of elastic fiber assembly. ${ }^{18}$

In spite of recent advances, the biology of fibrillins and related molecules, for example, the extent of the redundancy between molecules of the LTBP-fibrillin superfamily, remains incompletely understood. Recently LTBP2 null mutations were reported in primary congenital glaucoma (PCG). ${ }^{19,20}$ We here report biallelic null LTBP2 mutations in patients with eye features consisting primarily of microspherophakia, ectopia lentis, megalocornea, and myopia, and some features of MFS in older children.

\section{PATIENTS}

\section{Family 1}

The proband (VI.4), a boy, was first examined in the ophthalmology clinic at 18 months of age because of impaired vision. Spherophakia with infero-nasal displacement of both lenses was observed. The cornea was abnormally large (14-mm diameter in both eyes), with flat irides and iridodonesis. There was no nystagmus, and the eye pressure was normal (16 $\mathrm{mm} \mathrm{Hg}$ in both eyes). There was axial myopia (Right Eye (RE) $26.1 \mathrm{~mm}$, Left Eye (LE) $27.2 \mathrm{~mm}$ at 6 years). Bilateral phakophagia was performed at age 2 years for the lens luxation (Figure 1d). Retinal detachment presented in both eyes $4-5$ years later and ocular hypertension developed (up to $30 \mathrm{~mm} \mathrm{Hg}$ ). A proband's sister (VI.7) was referred at age 10 months because of megalocornea. She had good fixation and no nystagmus, but megalocornea (14.5-mm diameter), spherophakia, iridodonesis, and axial myopia were present. She had very deep anterior chamber, and miotic oval pupil with no well-defined border. Eye pressure was normal. Biometry performed at the age of 5 years showed an increased axial length of the globe (RE $27.0 \mathrm{~mm}$, LE $28.1 \mathrm{~mm}$ ), a deep anterior chamber (RE $3.9 \mathrm{~mm}, \mathrm{LE} 4.4 \mathrm{~mm}$ ), and increased lens diameter (RE $5.5 \mathrm{~mm}$, LE $5.6 \mathrm{~mm}$ ). Posterior lens dislocation was complete at the age of 6 years (Figure 1b). Ocular hypertony was noticed at the age of 8 years. The third affected child in this family (VI.8), another sister of the proband, had good fixation and no nystagmus, but presented at the age of 18 months with axial myopia, megalocornea (14.5-mm diameter) (Figure 1c), iridodonesis, iris anomalies (anterior synechiae and iris mamillations), deep anterior chambers, and spherophakia with no displacement of the lenses. Eye pressure was normal. At 5 years of age intermittent anterior dislocation of the lens in the left eye was observed, causing acute glaucoma crises and the left lens was extracted in emergency. Left retinal detachment developed a few weeks after. Progressive posterior luxation of the right lens, and bilateral ocular hypertony was observed.
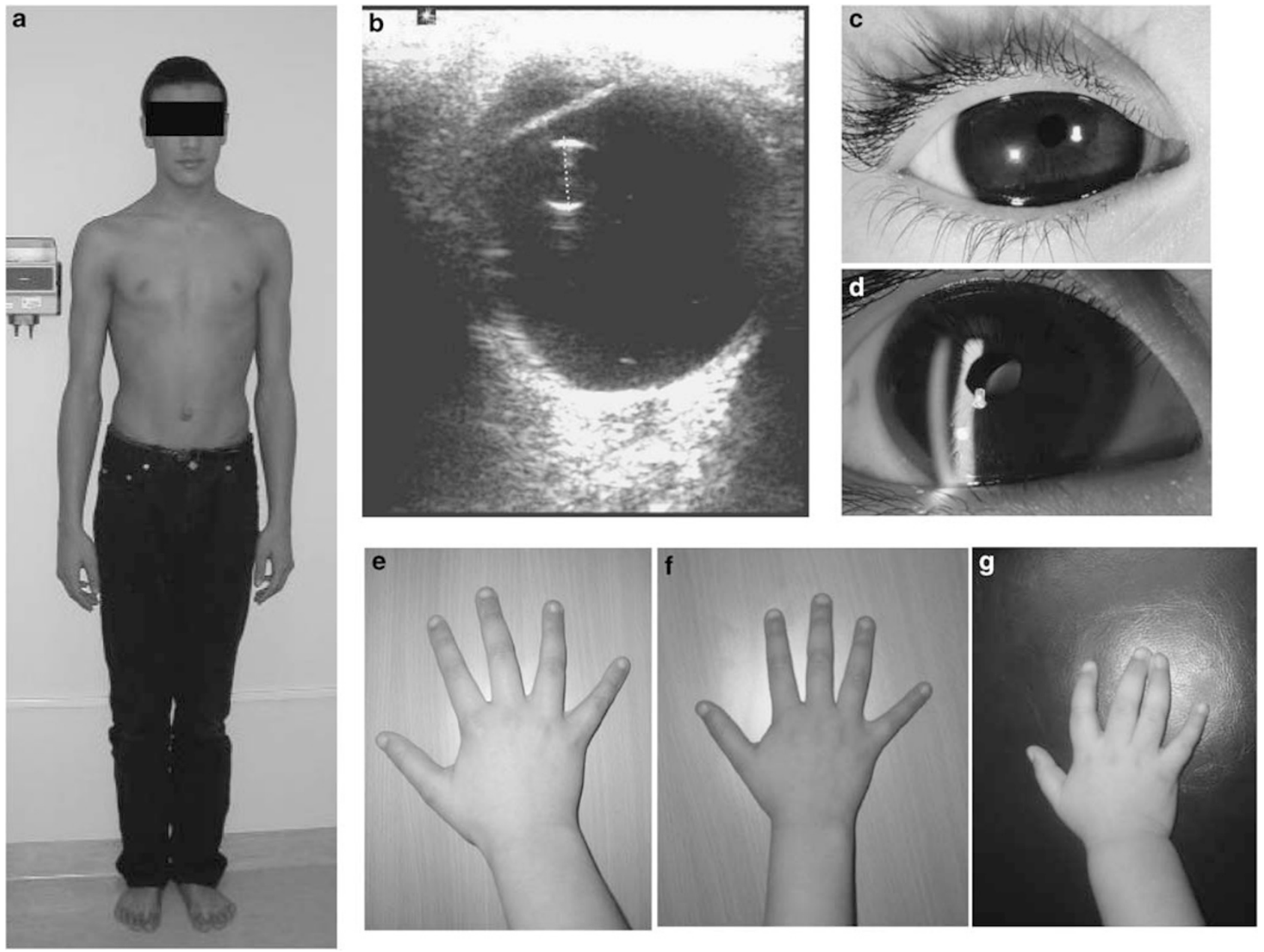

Figure 1 Clinical findings. (a) Proband (VI.4) of the first family, whose height was $184 \mathrm{~cm}$ and arm span $204 \mathrm{~cm}$ at the age of 14 years. (b) Ultrasound scan of the eye of patient VI.7 showing complete posterior luxation of the lens, as well as increased sphericity and short size of the lens. (c) The eye of the younger affected daughter (VI.8) of family 1 showing megalocornea. (d) Eye of proband (V4) showing inferonasal dislocation of the lens. (e-g) Hands of patients VI.7 and VI.8 from the first family and of proband from second family. 
Table 1 Clinical parameters

\begin{tabular}{|c|c|c|c|c|}
\hline Clinical features & Family I; VI.4 & Family I; VI.7 & Family I; VI.8 & Family II \\
\hline Lens dislocation & Nasal/nasal & Inferonasal/inferonasal & Inferonasal/inferonasal & Anterosuperior/anterosuperior \\
\hline Axial length of eye $(\mathrm{mm})$ & $26.1 / 27.2$ & $27.0 / 28.1$ & $22.0 / 24.0$ & $21.91 / 22.5$ \\
\hline Retinal detachment & $+/+$ & $-1-$ & $-1+$ & $-1-$ \\
\hline Glaucoma (age at onset) & 6 years & 8 years & 5 years & No glaucoma at 3 years \\
\hline $\mathrm{U} / \mathrm{L}$ or $\mathrm{SH} / \mathrm{H}^{\mathrm{b}}$ & $-2 S D$ & $+0.5 \mathrm{SD}$ & $-1 \mathrm{SD}$ & $-1 \mathrm{SD}$ \\
\hline High-arched palate & + & + & + & + \\
\hline Aortic root ectasia & - & - & - & - \\
\hline Cardiac valvular anomalies & - & - & - & - \\
\hline Cognitive defect (IQ) & At limit (75) & Mild (68) & Not evaluated, normal development & Not evaluated, normal development \\
\hline
\end{tabular}

aThe child was not compliant to test visual acuity, but she looked objects at $10 \mathrm{cms}$ of her eyes before lens was extracted.

bU/L: Upper/Lower body ratio; SH/H: Sitting Height/Height.

The family was referred to our Genetics department when the proband was 14 years of age. The parents were first cousins of Moroccan descent. The proband's height was $184 \mathrm{~cm}$, with an increased arm span of $204 \mathrm{~cm}$, and a decreased upper to lower body ratio $(83 \mathrm{~cm} / 101 \mathrm{~cm})$ (Figure $1 \mathrm{a})$. The hands were normal, with relatively long fingers (Figure 1e and f), albeit no frank arachnodactyly as the Walker sign (wrist sign) and Steinberg sign (thumb sign) were not present. The face was elongated. The palate was high-arched. The upper-to-lower body ratio was normal in both sisters with similar ocular abnormalities. The skin was normal on physical examination, with no striae distensae. The teeth and gums were unremarkable. The parents presented no features of MFS or Weill-Marchesani syndrome. Ophthalmological evaluation was normal in both parents and in the other six siblings. It is interesting to note that the proband and the older affected sister was in a special education school, and their IQ were just at or below normal (75 and 68, respectively). The younger affected sister had no systematic evaluation of mental capacities because psychomotor development and education were normal. Intelligence was normal in all other members of the family. A heart ultrasound showed a normal heart with normal valves and a normal aortic root in the proband and both affected sisters. Respiratory function tests were normal in the proband. No increased level of homocystine was found in the urine. A skin biopsy was performed for fibroblast culture. Marfan syndrome was considered and direct analysis of the FBN1 gene was performed, but no mutation was identified. The clinical parameters are shown in Table 1.

\section{Family 2}

We examined a second family of Macedonian descent with two children, one boy in good health and one affected girl. She was referred at age 26 months because of megalocornea. On ophthalmic examination the diameter of her corneas was $14 \mathrm{~mm}$ bilaterally. Slit lamp examination revealed a few patches of iris atrophy with iris transillumination and a very flat anterior chamber. The latter was associated with an anterior and superior dislocation of the lens. The lenses were abnormally round (spherophakia). The fundus and the eye pressure were normal. Biometry showed normal axial length of $21.91 \mathrm{~mm}$ (RE) and $22.5 \mathrm{~mm}$ (LE) (normal values for this age $21.63 \mathrm{~mm} \pm 0.77$ ). Keratometry was within normal range (mean of $42.5 \mathrm{D}$ RE and $43.37 \mathrm{D}$ LE). Her refraction was hypermetropic with astigmatism in the right $+16\left(+7135^{\circ}\right)$ and in the left eye $+12\left(+745^{\circ}\right)$. An iridectomy was performed but the anterior chamber remained very shallow. A clear lens was extracted to prevent acute angle closure glaucoma and to improve vision. At the age of 3 years, so far she did not develop glaucoma. At $2 \frac{1}{2} 2$ years, her arm span was $87 \mathrm{~cm}$, with a height of $88 \mathrm{~cm}$ and a sitting height of $51 \mathrm{~cm}$, giving normal arm span/height ratio and sitting height/height ratio for age. The hands were unremarkable (Figure $1 \mathrm{~g}$ ). The face was normal. The palate was high-arched. The skin was normal, with no striae distensae. The teeth and gums were unremarkable. Psychomotor development was normal (sitting at 5 months, walking at 1 year, speaking two languages). A heart ultrasound showed a normal heart with normal valves and a normal aortic root. The clinical parameters are shown in Table 1.

\section{METHODS}

A peripheral blood sample was obtained for genetic analysis with informed consent from the parents, and from the patients and unaffected siblings when old enough to consent, according to our study protocol as approved by the ethical committee of ULB - Hôpital Erasme. Control DNA subjects from Moroccan and Caucasian origin were recruited from our Genetic Centre and from a cohort of volunteers recruited in the Department of Occupational Medicine of our institution. Leukocyte DNA was extracted by the standard phenol-chloroform method. ${ }^{21}$ DNA samples were purified using the QiaAmp DNA microkit and resuspended in Tris- $\mathrm{HCl}$ buffer. A genome-wide search for homozygosity was performed in the affected siblings of family 1 using an Affymetrix $10 \mathrm{~K}$ GeneChip microarrays of 10000 autosomal single nucleotide polymorphisms (Affymetrix UK Ltd, High Wycombe, UK) following the instructions of the manufacturer. Regions of homozygosity were delineated using the Excludear algorithm. ${ }^{22}$ In chromosomal regions with apparent homozygosity by descent, additional microsatellites were genotyped in individual subjects. Marker order was obtained from the Cooperative Human Linkage Center genetic map (http://gai.nci.nih.gov/CHLC/) and from the University of California at Santa Cruz (UCSC) physical map (http://genome. ucsc.edu/cgi-bin/hgGateway). DNA was amplified by PCR using $15 \mathrm{ng}$ of DNA from each individual in a final volume of $15 \mu \mathrm{l}$, followed by polyacrylamide gel electrophoresis and silver staining. A multipoint LOD score was computed using the MAPMAKER/HOMOZ software postulating a gene frequency of 0.005 and marker allele frequencies as observed in a series of control subjects, with a minimal minor allele frequency of 0.10 . The 36 coding exons and intron/exon junctions of LTBP2 were amplified using primers designed by the ExonPrimer Perl script engine (Munchen, Germany). Polymerase chain reaction products were purified using the Exosap-IT kit (USB, Cleveland, OH, USA) and directly sequenced by the Big Dye Terminator 
cycle sequencing kit v2 (Applied Biosystems, Foster City, CA, USA). Sequencing products were purified on Egde Biosystems Performa DTR Ultra 96-Well Plate Kit columns (Sopachem, Eke, Belgium) and analyzed on a 3130 Genetic Analyzer sequencing machine (Applied Biosystems). Sequences were inspected in silico for mutations using the SeqSape software version 2.0 (Applied Biosystems).

Skin fibroblasts of the proband were primary cultured using standard procedures. The cells were then lyzed in TRIzol reagent (Invitrogen, Carlsbad, CA, USA) and the cellular RNA was extracted by a conventional phenolchloroform method. The RNA was reverse-transcribed in single-stranded cDNA using the SuperScript First Strand Synthesis System kit for RT-PCR (Invitrogen). The second cDNA strand was synthesized and a PCR was performed using Taq DNA polymerase (Invitrogen), to amplify a 625-bp fragment of LTBP2 cDNA with direct primer situated in exon 7 and reverse primer in exon 12 (Direct primer sequence: ACAGCAACAACATCCCTGC; Reverse primer sequence: GCGTAGGTGTAGCCGTGG). The amplified products were detected by running a $1 \%$ agarose gel with Lambda DNA/Hind III and PhiX 174 DNA/HaeIII Mix molecular weight marker (NEB). The gels were stained in ethidium bromide, observed under UV transilluminator and then photographed.

\section{RESULTS}

\section{Family 1}

The initial homozygosity mapping performed in the three patients of the first family using the $10 \mathrm{~K}$ GeneChip identified two large homozygous segments, on $9 \mathrm{q} 31.3-\mathrm{q} 33.2$ and on $14 \mathrm{q} 23.3-24.3$. We re-analyzed these regions with microsatellite markers in all family members. This showed that the 9q31.3-q33.2 region was also homozygous in an asymptomatic brother, with normal habitus and in whom ophthalmologic examination was normal. Assuming complete penetrance of the trait, we hence rejected linkage to chromosome 9 and considered the $14 \mathrm{q}$ region only (Figure 2). A maximal multipoint LOD score of 2.87 at D14S1002 was calculated using the MAPMAKER/HOMOZ software. The 14q linkage segment contained 199 annotated genes. Computer-assisted in silico inspection using the Endeavour software program, ${ }^{23}$ indicated $L T B P 2$ gene as a strong candidate. Direct sequencing of LTBP2 identified a homozygous mutation in the proband and both affected sisters, consisting of a

।

II

III

.

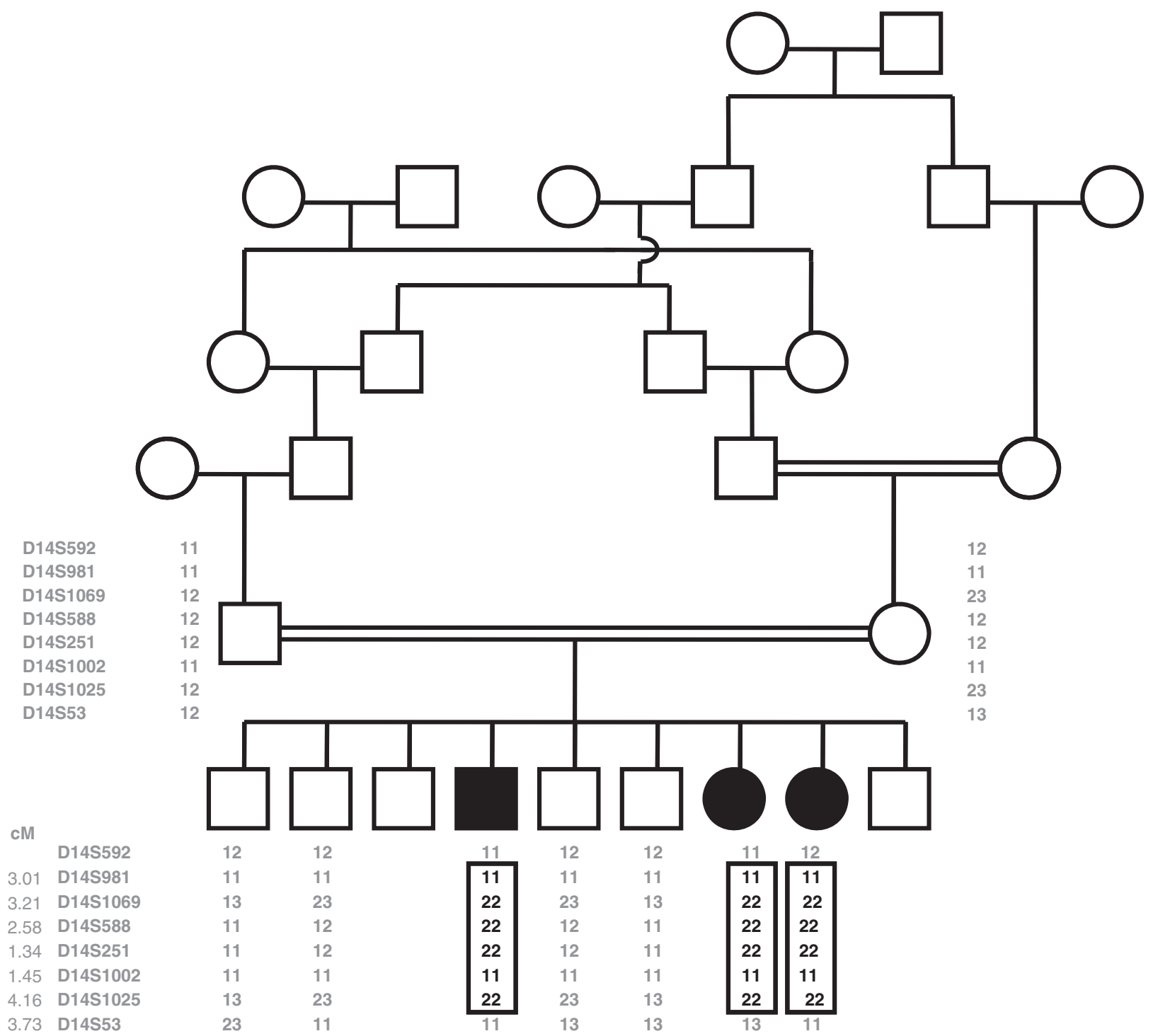

Figure 2 Linkage analysis in family 1. The parents were second cousins. The 14q linkage region was initially identified by a genome-wide $10 \mathrm{~K}$ GeneChip microarray of SNPs in three affected siblings, then further analyzed by microsatellite polymorphisms in affected and unaffected siblings and parents, as shown. A single linkage region was found where all patients and no unaffected siblings were homozygous, yielding a maximal multipoint LOD score of 2.87 at $\mathrm{D} 14 \mathrm{~S} 1002$. 

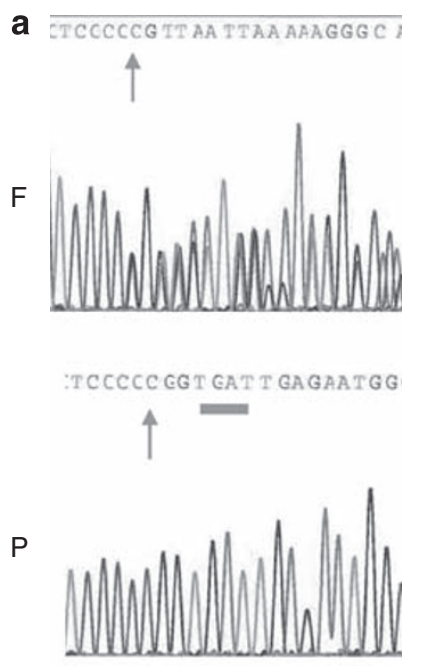

$\overline{\text { TCCCCGGT GAI TGAG AATGGC }}$

C

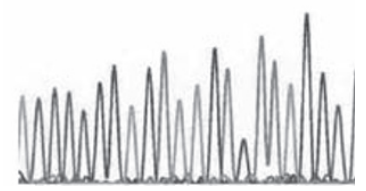

b

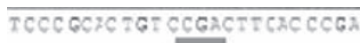

$\mathrm{F}$

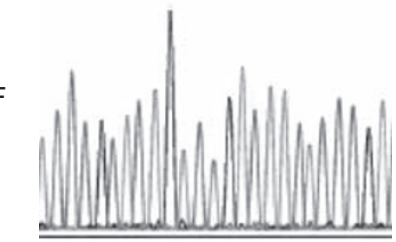

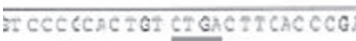

$1 \mathrm{nn}$

$\mathrm{P}$

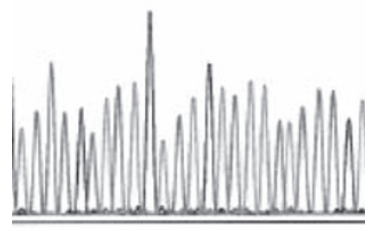

iaCCCCACTGZCCGACTICACCCGA

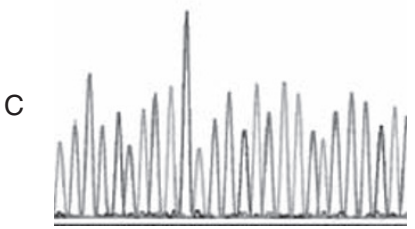

c

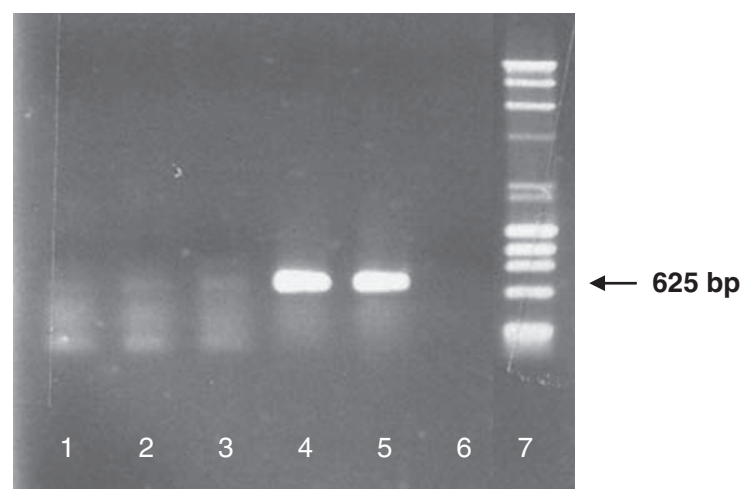

Figure 3 LTBP2 gene sequencing and mRNA analysis. (a) Family 1. Top to bottom, direct sequencing of genomic DNA from father, proband, and an ethnically matched unrelated control, showing c.1796_1797insC (p.Val600GlyfsX2) mutation in exon 9 of LTBP2, heterozygous in father and homozygous in proband. (b) Family 2. Top to bottom, direct sequencing of genomic DNA from father, proband, and an ethnically matched unrelated control, showing c.895C > T (p.Arg299X) mutation in exon 4 of LTBP2, heterozygous in father and homozygous in proband. (c) Analysis of LTBP2 transcript in cultured fibroblasts using primers situated in exons 7 and 12. 1-3: RT-PCR of three aliquots of mRNA extracted from fibroblasts culture of Family 1's proband; 4-5: RT-PCR of two unrelated control fibroblast cultures, showing a band of expected size $(625 \mathrm{bp})$; 6: Negative control with H2O used in place of mRNA template; 7: Lambda DNA/Hind III and PhiX 174 DNA/HaellI Mix molecular weight marker.

1-bp insertion in exon 9, causing a frameshift responsible for a premature stop codon (c.1796dupC; p.Val600GlyfsX2). Both parents were heterozygous for the mutation, and no unaffected sibling was homozygous. The mutation was not found in 100 Moroccan control subjects (Figure 3). A fragment of the LTBP2 mRNA encompassing exons 7 through 12, that is, surrounding the frameshift mutation and premature stop codon in exon 9, was amplified from the proband's cultured skin fibroblasts by RT-PCR. A band of the expected size $(625 \mathrm{bp})$ was observed in unrelated controls. This band was absent in the patient, and no other band was detected (Figure 3). This result is consistent with nonsense-mediated mRNA decay.

\section{Family 2}

In the second family, direct sequencing of the LTBP2 gene identified a homozygous nonsense mutation in exon 4 (c.895C > T; p.Arg299X). Both parents were heterozygous carriers of the mutation, which was absent from 100 Caucasian controls (Figure 3).

\section{DISCUSSION}

We found biallelic LTBP2 null mutations in two unrelated families ascertained via the ophthalmology clinic. Megalocornea and microspherophakia with early ectopia lentis probably secondary to the small round lens appears to be the most specific findings in these families. Megalocornea and microspherophakia may be encountered in congenital Marfan syndrome, ${ }^{3,4}$ but the incidence in the total Marfan population is probably very low. Megalocornea was present in all LTBP2-mutated patients reported to date. ${ }^{19,20}$ However, the patients in this report presented no corneal clouding, contrary to the patients previously reported. ${ }^{19,20}$ Megalocornea with microspherophakia is a condition that must be considered in the differential diagnosis of PCG. ${ }^{24}$ Glaucoma may be congenital or may develop during childhood. The pathogenesis of the glaucoma is incompletely understood and normal gonioscopy can be found with megalocornea. ${ }^{25}$ Recurrent attacks of pupillary block may lead to the development of glaucoma at a very young age and may therefore be clinically misdiagnosed as PCG. Glaucoma in our patients developed later than previously reported: ${ }^{19,20}$ after the age of 5 years in Family 1, not present at 3 years in Family 2. The intraocular pressures were normal in all patients at initial evaluation. Glaucoma was thus secondary, not primary nor congenital. Microspherophakia was identified by biometry and by biomicroscopy (after pupil dilatation the edge (equator) of each crystalline lens was easily visible with slit lamp). Spherophakia and iridodonesis were already present at the age of 10 months, and dislocation developed in all 8 eyes beginning as early as the age of 2 years, with inferonasal and backward displacement, producing an image of broken zonule, as in homocystinuria in Family 1. The displacement was anterosuperior in Family 2. Complete posterior lens dislocation occurred in 3 of 8 eyes.

Some signs of increased growth of the long bones were seen in older patients. All had a high-arched palate. The proband in Family 1 had 
a height of $184 \mathrm{~cm}$ at the age of 14 years, which contrasted with the heights of his mother and father $(165$ and $172 \mathrm{~cm})$, a slender habitus, and an arm span of $204 \mathrm{~cm}$, giving an arm span/height ratio of 1.11 (Normal $<1.05)$. An arm span greater than height was present in his 8 -year-old sister, perhaps indicating a progression of increased linear growth over time. Thus, some MFS features could be part of the LTBP2 defect-associated phenotypic spectrum. Although ectopia lentis and marfanoid habitus were found in some of the recently reported patients, including tall stature, an arm span greater than height, and arachnodactyly, no detailed data were provided. ${ }^{19,20}$ Interestingly, none of the patients had cardiovascular symptoms, and all had a normal cardiac ultrasound. The skin did not show striae distensae. Therefore, we found no evidence of intense elastolysis and defective tissue repair as reported in MFS. ${ }^{9}$ This is in line with in vitro data showing that LTBP2 is not able to form a covalent complex with LAP.TGF $\beta 1 .^{15}$

LTBP2 is expressed in the trabecular meshwork and ciliary processes, with a high expression in the lens capsule/lens epithelium layer in the adult mouse, cow's eye ${ }^{19}$ and in the human eye. ${ }^{20}$

Isolated, non-syndromic familial ectopia lentis has been reported as an autosomal recessive trait associated with ADAMTSL4 mutations, ${ }^{26}$ or as an autosomal-dominant trait-associated with FBN1 mutations. ${ }^{27}$ Ectopia lentis is reported in MFS, and in Weill-Marchesani syndrome (MIM277600), a rare connective tissue disorder characterized by short stature, brachydactyly, joint stiffness, and lens abnormalities, associated with ADAMTSL10 gene mutations. ${ }^{28}$ It is also a feature of homocystinuria (MIM236200) and of sulfocysteinuria (MIM272300). The two latter disorders suggest that ectopia lentis may result from a chemical alteration of disulfide bonds in the microfibrillar network of the ocular zonule that are essential for holding the lens in a dynamic equilibrium. Taken together, we speculate that LTBP2 and FBN1 might have partially redundant structural functions in the zonule, with additional modelling by ADAMTS10 and possibly other proteases.

The zonule and the periodontal ligament share the feature of being rich in microfibrils and devoid of elastin. ${ }^{29}$ Therefore, we thoroughly examined the teeth, periodontal sulci and gingivae in our patients, but found no abnormality.

The older two affected sibs in family 1 had an IQ just at or below the normal range. It is presently unclear whether mental or developmental delay co-segregates with the LTBP2 defect, as the third affected child in family 1 as well as the patient in family 2 had normal milestones.

Mutation c.1796dupC (p.Val600GlyfsX2) found in Moroccan Family 1 affects the hybrid domain and has not been reported before. No Moroccan patients with LTBP2 mutations have been reported to date. ${ }^{19,20}$ Mutation c.895C $>$ T (p.Arg299X) found in family 2 was recently described in PCG patients from Pakistan and in European Gypsies. ${ }^{19}$ The parents in Family 2 are Macedonian Gypsies, not known to be related. The region where they originated from (Skopje and Chtip) encompasses gypsy minorities (http://en.wikipedia.org/ wiki/Roma_in_the_Republic_of_Macedonia). This finding suggests that the p.R299X LTBP2 defect may represent an ancient founder mutation and may present either as PCG or as megalocornea, spherophakia, and secondary glaucoma.

Both mutations are predicted to produce truncated proteins. To date, 5 of the 6 LTBP2 mutations reported, and the mutation p.Val600GlyfsX2 affecting the hybrid domain, are located in the amino-terminal part of the protein, interfering with both fibrillin-1 and fibulin-5 binding. ${ }^{19,20}$ So far, only one mutation has been described affecting only its carboxy-terminal domain, suggesting that interference with fibulin-5 alone can be deleterious. ${ }^{20}$ The relatively mild phenotype of these null mutations in man contrasts with in utero lethality of ltbp2-1- mice, that die between embryonic day 3.5 (E3.5) and E6.5. ${ }^{29}$ LTBP2 is a very large, highly repetitive molecule, and many of its modules are encoded by single, in-frame exons. ${ }^{11}$ No evidence of exon skipping was found in family 1 (Figure 3).

In summary, here we report homozygous null mutations of LTBP2 in two unrelated families from different ethnic backgrounds, associated with an autosomal recessive syndrome of microspherophakia and progressive ectopia lentis, myopia and no primary congenital glaucoma, as well as some Marfan features in the older patient. Glaucoma developed after the age of 5 years in the first family. We suggest that intraocular pressure should be followed-up in young children with megalocornea, spherophakia and/or lens dislocation. LTBP2 gene analysis should be offered to these patients.

\section{CONFLICT OF INTEREST}

The authors declare no conflict of interest.

\section{ACKNOWLEDGEMENTS}

We thank JF Laes, Biovallee, Gosselies, Belgium, for initial analysis of the $10 \mathrm{~K}$ Affymetrix GeneChips, Z Ajarchouh for expert technical work, and G Vassart for support. We are indebted to P Coucke and B Loeys, Gent, for FBN1 gene sequencing and useful discussion. We thank V Cormier-Daire, Paris, for interest and discussion. JD is a fellow of the Belgian Kids' Fund and of the Erasme Fund for Scientific Research. MJA is supported by Grant no. 3.4593.07 of the Fonds de la Recherche Scientifique Médicale (FRSM), Belgium.

1 De Paepe A, Devereux RB, Dietz HC, Hennekam RC, Pyeritz RE: Revised diagnostic criteria for the Marfan syndrome. Am J Med Genet 1996; 62: 417-426.

2 Faivre L, Masurel-Paulet A, Collod-Beroud G et al: Clinical and molecular study of 320 children with Marfan syndrome and related type I fibrillinopathies in a series of 1009 probands with pathogenic FBN1 mutations. Pediatrics 2009; 123: 391-398.

3 Meire FM, Delleman WJ, Bleeker-Wagemakers EM: Ocular manifestations of congenital Marfan syndrome with contractures (CMC syndrome). Ophthalmic Paediatr Genet 1991; 12: 1-9.

4 Meire FM, Van EJ, Hanssens M: Congenital Marfan syndrome with contractures. A clinicopathological report. Bull Soc Belge Ophtalmol 1992; 245: 91-97.

5 Ramirez F, Dietz HC: Fibrillin-rich microfibrils: structural determinants of morphogenetic and homeostatic events. J Cell Physiol 2007; 213: 326-330.

6 Dietz HC, Cutting GR, Pyeritz RE et al: Marfan syndrome caused by a recurrent de novo missense mutation in the fibrillin gene. Nature 1991; 352: 337-339.

7 Isogai Z, Ono RN, Ushiro S et al: Latent transforming growth factor beta-binding protein 1 interacts with fibrillin and is a microfibril-associated protein. J Biol Chem 2003; 278: 2750-2757.

8 Gregory KE, Ono RN, Charbonneau NL et al: The prodomain of BMP-7 targets the BMP-7 complex to the extracellular matrix. J Biol Chem 2005; 280: 27970-27980.

9 Neptune ER, Frischmeyer PA, Arking DE et al: Dysregulation of TGF-beta activation contributes to pathogenesis in Marfan syndrome. Nat Genet 2003; 33: 407-411.

10 Habashi JP, Judge DP, Holm TM et al: Losartan, an AT1 antagonist, prevents aortic aneurysm in a mouse model of Marfan syndrome. Science 2006; 312: 117-121.

11 Massague J: The TGF-beta family of growth and differentiation factors. Cell 1987; 49: 437-438.

12 Robinson PN, Arteaga-Solis E, Baldock C et al: The molecular genetics of Marfan syndrome and related disorders. J Med Genet 2006; 43: 769-787.

13 Gleizes PE, Beavis RC, Mazzieri R, Shen B, Rifkin DB: Identification and characterization of an eight-cysteine repeat of the latent transforming growth factor-beta binding protein- 1 that mediates bonding to the latent transforming growth factor-beta1. J Biol Chem 1996; 271: 29891-29896.

14 Saharinen J, Taipale J, Keski-Oja J: Association of the small latent transforming growth factor-beta with an eight cysteine repeat of its binding protein LTBP-1. EMBO J 1996; 15: 245-253.

15 Saharinen J, Keski-Oja J: Specific sequence motif of 8-Cys repeats of TGF-beta binding proteins, LTBPs, creates a hydrophobic interaction surface for binding of small latent TGF-beta. Mol Biol Cell 2000; 11: 2691-2704.

16 Reinhardt DP, Gambee JE, Ono RN, Bachinger HP, Sakai LY: Initial steps in assembly of microfibrils. Formation of disulfide-cross-linked multimers containing fibrillin-1. J Biol Chem 2000; 275: 2205-2210.

17 Hirani R, Hanssen E, Gibson MA: LTBP-2 specifically interacts with the amino-terminal region of fibrillin-1 and competes with LTBP-1 for binding to this microfibrillar protein. Matrix Biol 2007; 26: 213-223. 
18 Hirai M, Horiguchi M, Ohbayashi T, Kita T, Chien KR, Nakamura T: Latent TGF-betabinding protein 2 binds to DANCE/fibulin- 5 and regulates elastic fiber assembly. EMBO J 2007; 26: 3283-3295.

19 Ali M, McKibbin M, Booth A et al: Null mutations in LTBP2 cause primary congenital glaucoma. Am J Hum Genet 2009; 84: 664-671.

20 Narooie-Nejad M, Paylakhi SH, Shojaee S et al: Loss of function mutations in the gene encoding latent transforming growth factor beta binding protein 2, LTBP2, cause primary congenital glaucoma. Hum Mol Genet 2009; 18: 3969-3977.

21 Sambrook J, Fritsch EF, Maniatis T: Molecular Cloning: A Laboratory Manual, 2nd edn. Cold Spring Harbor, NY: Cold Spring Harbor Laboratory, 1989.

22 Woods CG, Valente EM, Bond J, Roberts E: A new method for autozygosity mapping using single nucleotide polymorphisms (SNPs) and EXCLUDEAR. J Med Genet 2004; 41: e101.

23 Aerts S, Lambrechts D, Maity S et al: Gene prioritization through genomic data fusion. Nat Biotechnol 2006; 24: 537-544.

24 Roche O, Dureau P, Uteza Y, Dufier JL: Congenital megalocornea. J Fr Ophtalmol 2002; 25: 312-318.
25 Meire FM: Megalocornea. Clinical and genetic aspects. Doc Ophthalmol 1994; 87: 1-121. 26 Ahram D, Sato TS, Kohilan A et al: A homozygous mutation in ADAMTSL4 causes autosomal-recessive isolated ectopia lentis. Am J Hum Genet 2009; 84: 274-278.

27 Ades LC, Holman KJ, Brett MS, Edwards MJ, Bennetts B: Ectopia lentis phenotypes and the FBN1 gene. Am J Med Genet A 2004; 126A: 284-289.

28 Dagoneau N, Benoist-Lasselin C, Huber C et al: ADAMTS10 mutations in autosomal recessive Weill-Marchesani syndrome. Am J Hum Genet 2004; 75: 801-806.

29 Shipley JM, Mecham RP, Maus E et al: Developmental expression of latent transforming growth factor beta binding protein 2 and its requirement early in mouse development. Mol Cell Biol 2000; 20: 4879-4887.

(c) This work is licensed under the Creative Commons Attribution-NonCommercial-No Derivative Works 3.0 Unported License. To view a copy of this license, visit http:// creativecommons.org/licenses/by-nc-nd/3.0/ 